K. KOMIYA

KODAI MATH. J.

18 (1995), $344-350$

\title{
EQUIVARIANT CRITICAL POINT THEORY AND SET-VALUED GENERA
}

Dedicated to Professor Seiya Sasao on his 60th birthday

\author{
KATSUHIRO KOMIYA
}

\section{Introduction}

Throughout this paper $G$ denotes a compact Lie group. For a point $x$ of a $G$-space $X$ we set

$$
G x:=\{g x \in X \mid g \in G\}, \text { and } G_{x}:=\{g \in G \mid g x=x\} .
$$

$G x$ is called the orbit through $x$, and $G_{x}$ the isotropy subgroup at $x$. Its conjugacy class $\left(G_{x}\right)$ is called the orbit type (or isotropy type) of $x$. We denote by $g(X)$ the set of all orbit types on $X$.

Let $M$ be a closed $G$-manifold, or more generally a paracompact Banach $G$-manifold of class at least $C^{1}$, i.e., $M$ is a paracompact $C^{1}$ Banach manifold and $G$ acts differentiably by diffeomorphisms. Let $f: M \rightarrow \boldsymbol{R}$ be a $C^{1} G$-function, i.e., $f$ is of class $C^{1}$ and satisfies $f(g x)=f(x)$ for all $x \in M$ and $g \in G$. If $x \in M$ is a critical point of $f$, i.e., $d f_{x}=0$, then its orbit $G x$ is called a critical orbit and its orbit type $\left(G_{x}\right)$ is called a critical orbit type. Let $K=\left\{x \in M \mid d f_{x}=0\right\}$ be the critical point set of $f$, and set $K_{c}=K \cap f^{-1}(c)$ and $M_{c}=\{x \in M \mid f(x) \leqq c\}$ for any $c \in \boldsymbol{R}$.

The number of critical points alone has been discussed in the nonequivariant setting. We should, however in the equivariant setting, discuss the types of critical orbits as well as the number of them. There also arises a new problem to estimate the number of critical orbit types. In this paper we will define the set-valued genus and the numerical genus of a $G$-space, and obtain some inequalities for the genus and the number of critical values and orbit types for $G$-functions.

Let $\mathcal{A}$ be the set of $G$-homeomorphism classes of compact $G$-ANR's ( $G$ equivariant Absolute Neighborhood Retract). For a $G$-space $X$ we define a subset $\alpha(X)$ of $\mathcal{A}$ to be the set of those $S \in \mathcal{A}$ for which there is a $G$-map $X \rightarrow S$. We call $\alpha(X)$ the set-valued genus of $X$. For two subsets $\alpha_{1}$ and $\alpha_{2}$ of $\mathcal{A}$, we define

$$
\alpha_{1} * \alpha_{2}:=\left\{S * T \mid S \in \alpha_{1}, T \in \alpha_{2}\right\} .
$$

Received February 21, 1994. 
where $S * T$ denotes the join of $S$ and $T$.

Suppose $f: M \rightarrow \boldsymbol{R}$ is bounded below and satisfies the Palais-Smale condition or the conditions $\left(\mathrm{R}_{0}\right)-\left(\mathrm{R}_{2}\right)$ stated below. If the critical values of $f$ are $c_{1}, \cdots$, $c_{m}$, then we will obtain

$$
\alpha\left(K_{c_{1}}\right) * \cdots * \alpha\left(K_{c_{m}}\right) \leqq \alpha(M) .
$$

For a $G$-space $X$ and a subset $\mathcal{S}$ of $\mathcal{A}$, we define $\beta_{S}(X)$ to be the smallest number $n$ such that $A_{1} * \cdots * A_{n} \in \alpha(X)$ for $A_{1}, \cdots, A_{n} \in \mathcal{S}$. We call $\beta_{S}(X)$ the numerical $S$-genus of $X$. Since a homogeneous space $G / H$ with $H$ a closed subgroup of $G$ is a $G$-ANR, we may take as $\mathcal{S}$ the following subset $\mathscr{H}$ or $\mathcal{O}(X)$ of $A$,

$$
\begin{gathered}
\mathscr{H}=\{G / H \mid H \text { is a proper closed subgroup of } G\}, \\
\mathcal{O}(X)=\{G / H \mid(H) \in \mathcal{G}(X)\} .
\end{gathered}
$$

Then under some conditions on $f: M \rightarrow R$ we will obtain from (0.1),

and

$$
\beta_{\mathscr{T}}(M) \leqq \# f(K) \cdot \# \mathcal{I}(K),
$$

$$
\beta_{\mathcal{O}(M)}(M) \leqq \# f(K) \cdot \# \mathcal{G}(K),
$$

(see Theorem 3.1 below), where $\# f(K)$ is the number of critical values and $\# \mathscr{I}(K)$ the number of critical orbit types.

The author thanks the referee for his or her comments which lead to the improvement of the earlier draft of this paper.

\section{Set-valued genera}

A $G$-ANR is a metrizable $G$-space $S$ such that every $G$-map $Z \rightarrow S$ from a $G$-invariant closed subspace $Z$ of a metrizable $G$-space $Y$ into $S$ has a $G$-extension $U \rightarrow S$ to a $G$-neighborhood $U$ of $Z$ in $Y$. Any point of the join $S * T$ of two $G$-ANS's $S$ and $T$ is represented by the form $[(\lambda, x),(\mu, y)]$ with the usual identification, where $x \in S, y \in T, 0 \leqq \lambda, \mu \leqq 1$ and $\lambda+\mu=1$. Then $S * T$ becomes a $G$-space via the action

$$
g[(\lambda, x),(\mu, y)]=[(\lambda, g x),(\mu, g y)]
$$

for any $g \in G$. Moreover $S * T$ is a $G$-ANR (see Murayama [4; Corollary 12.3]). This implies that the join $\alpha_{1} * \alpha_{2}$ of subsets $\alpha_{1}$ and $\alpha_{2}$ of $\mathcal{A}$ is also a subset of $\mathcal{A}$.

Convention 1.1. We make the following conventions concerning the empty set $\emptyset$ and the set-valued genus $\alpha(X)$ of a $G$-space $X$.

(i) The empty set $\emptyset$ is assumed to belong to $\mathcal{A}$.

(ii) If $X=\emptyset$ then we assume $\alpha(X)=\mathcal{A}$, and if $X \neq \emptyset$ then $\emptyset \notin \alpha(X)$.

(iii) $X * \emptyset=X=\emptyset * X$ for any space $X$. Then we see $\alpha \subseteq \alpha * \mathcal{A}$ for any subset 
$\alpha$ of $\mathcal{A}$, since $\emptyset \in \mathcal{A}$.

(iv) Considering $\emptyset$ as the empty subset of $\mathcal{A}, \alpha * \emptyset=\emptyset=\emptyset * \alpha$ for any subset $\alpha$ of $\mathcal{A}$.

The set-valued genus possesses the following simple properties.

Proposition 1.2. (i) Monotonicity: If there is a G-map $X \rightarrow Y$, then $\alpha(Y) \subseteq \alpha(X)$.

(ii) Subadditivity: If $X=A \cup B$ is a metrizable $G$-space and $A, B$ are $G$ invariant closed subsets of $X$, then $\alpha(A) * \alpha(B) \leqq \alpha(X)$.

(iii) Subcontinuity: Let $X$ be a metrizable G-space and $A$ a G-invariant closed subset of $X$. For any finite subset $I$ of $\alpha(A)$, there is a G-neighborhood $U_{\mathscr{F}}$ of $A$ in $X$ such that $\mathscr{F} \leqq \alpha(U) \leqq \alpha(A)$ for any $G$-neighborhood $U$ of $A$ with $U \subseteq U_{\mp}$.

Proof. (i) Easy.

(ii) Assume $\alpha(A) \neq \emptyset$ and $\alpha(B) \neq \emptyset$, and take $S \in \alpha(A)$ and $T \in \alpha(B)$. There are $G$-maps $\varphi: A \rightarrow S$ and $\phi: B \rightarrow T$. Since $S$ and $T$ are $G$-ANR's, we have their $G$-extensions $\tilde{\varphi}: N_{A} \rightarrow S$ and $\tilde{\phi}: N_{B} \rightarrow T$, where $N_{A}$ and $N_{B}$ are open $G$ neighborhood of $A$ and $B$ in $X$, respectively. There is a function $\lambda: X \rightarrow[0,1]$ such that $\lambda=0$ on $X-N_{A}$ and $\lambda=1$ on $X-N_{B}$. We may assume that $\lambda$ is $G$ invariant since we may integrate it over $G$ if necessary. We may define a $G$ map $\eta: X \rightarrow S * T$ by

$$
\eta(x)=[(\lambda(x), \tilde{\varphi}(x)),(1-\lambda(x), \tilde{\psi}(x))]
$$

for $x \in X$. Thus $S * T \in \alpha(X)$.

(iii) If $\mathscr{F}$ is empty, then $\mathscr{I} \subseteq \alpha(U) \subseteq \alpha(A)$ for all $G$-neighborhood $U$ of $A$. So assume $\mathscr{F} \neq \emptyset$, and take $S \in \mathscr{F}$ and a $G$-map $\varphi: A \rightarrow S$. Then $\varphi$ has a $G$ extension $U_{S} \rightarrow S$ to a $G$-neighborhood $U_{S}$ of $A$, and thus $S \in \alpha\left(U_{S}\right)$. Set $U_{q}$ : $=\bigcap_{S \in \mathscr{T}} U_{S}$. Then $U_{\mathscr{F}}$ is a $G$-neighborhood of $A$ since $\mathscr{T}$ is finite. For any $S \in \mathscr{F}$ and $G$-neighborhood $U$ of $A$ with $U \subseteq U_{\mathcal{F}}$, we see $\alpha\left(U_{S}\right) \subseteq \alpha\left(U_{\mathscr{T}}\right) \subseteq \alpha(U)$ by the monotonicity property. Thus $\mathscr{F} \leqq \bigcup_{S \in \mathcal{F}} \alpha\left(U_{S}\right) \subseteq \alpha(U)$.

\section{The genera of critical point sets}

Throughout this paper $M$ denotes a paracompact $C^{1}$ Banach $G$-manifold and $f: M \rightarrow \boldsymbol{R}$ a $C^{1} G$-function with the critical point set $K$. Consider the following conditions $\left(\mathrm{R}_{0}\right)-\left(\mathrm{R}_{2}\right)$ for $f: M \rightarrow \boldsymbol{R}$ at $c \in \boldsymbol{R}$ :

$\left(\mathrm{R}_{0}\right)$ There are an $\varepsilon>0$ and a $G-\operatorname{map} M_{c+\varepsilon} \rightarrow M_{c}$.

$\left(\mathrm{R}_{1}\right) K_{c}$ is compact.

$\left(\mathrm{R}_{2}\right)$ For every $G$-neighborhood $U$ of $K_{c}$ there are an $\varepsilon>0$ with $\varepsilon<1$ and a $G$-map $\varphi: M_{c+\varepsilon}-U \rightarrow M_{c-\varepsilon}$ (with $\varphi \mid M_{c-1}=$ id if $f$ is unbounded above and $c>$ $\sup f(K)$ ). 
The conditions $\left(R_{0}\right)-\left(R_{2}\right)$ are weaker than the conditions $\left(D_{0}\right)-\left(D_{2}\right)$ in ClappPuppe $[2 ; 3.1]$. As is shown in Clapp-Puppe $[2$; Appendix A] and KrawcewiczMarzantowicz [3; Lemma 1.9], the conditions $\left(\mathrm{R}_{1}\right)$ and $\left(\mathrm{R}_{2}\right)$ at any $c \in \boldsymbol{R}$ is a consequence of the Palais-Smale condition under suitable assumptions on differentiability and completeness. [2; Appendix A] shows that if $c$ is a regular value of $f$ then $\left(\mathrm{R}_{0}\right)$ is also a consequence of the Palais-Smale condition. Even if $c$ is not a regular value we can see that $\left(\mathrm{R}_{0}\right)$ follows from the Palais-Smale condition under the assumption $c$ is an isolated critical value.

LEMMA 2.1. Suppose that a $C^{1} G$-function $f: M \rightarrow \boldsymbol{R}$ satisfies $\left(\mathrm{R}_{1}\right)$ and $\left(\mathrm{R}_{2}\right)$ at c. For a given finite subset $\mathscr{F}$ of $\alpha\left(K_{c}\right)$ there is an $\varepsilon=\varepsilon(\mathscr{F})>0$ such that

$$
\alpha\left(M_{c-\varepsilon}\right) * \mathscr{F} \subseteq \alpha\left(M_{c+\varepsilon}\right) .
$$

In particular, if $K_{c}$ is empty then $\alpha\left(M_{c-\varepsilon}\right)=\alpha\left(M_{c+\varepsilon}\right)$.

Proof. By the assumption, $K_{c}$ is compact and thus closed. $M$ is metrizable by Palais [5; Corollary 3.4]. By the subcontinuity property of $\alpha$ there is a $G$-neighborhood $U_{\mathscr{F}}$ of $K_{c}$ in $M$ such that $\mathscr{F} \leqq \alpha(U) \subseteq \alpha\left(K_{c}\right)$ for any $G$-neighborhood $U$ of $K_{c}$ with $U \cong U_{\mathcal{I}}$. Take an open $G$-neighborhood $V$ of $K_{c}$ such that $\bar{V} \cong U_{\mathscr{F}}$, and take an $\varepsilon>0$ satisfying $\left(\mathrm{R}_{2}\right)$ for this $V$. Then we see

$$
\begin{aligned}
\alpha\left(M_{c+\varepsilon}\right) & =\alpha\left(\left(M_{c+\varepsilon}-V\right) \cup \bar{V}\right) \\
& \supseteq \alpha\left(M_{c+\varepsilon}-V\right) * \alpha(\bar{V}) \\
& \supseteq \alpha\left(M_{c-\varepsilon}\right) * \mathscr{F} .
\end{aligned}
$$

If $K_{c}$ is empty, then there are an inclusion map $M_{c-\varepsilon} \rightarrow M_{c+\varepsilon}$ and a $G-\operatorname{map} M_{c+\varepsilon} \rightarrow$ $M_{c-\varepsilon}$ by $\left(\mathrm{R}_{2}\right)$. Using the monotonicity property twice, we see $\alpha\left(M_{c-\varepsilon}\right)=\alpha\left(M_{c+\varepsilon}\right)$.

Consider an interval $(a, b]=\{t \in \boldsymbol{R} \mid a<t \leqq b\}$ for $-\infty<a<b \leqq \infty$. A $C^{1} G$ function $f: M \rightarrow \boldsymbol{R}$ is said to satisfy $\mathrm{R}(a, b)$ if $f$ satisfies $\left(\mathrm{R}_{0}\right)$ at $a$ and $\left(\mathrm{R}_{1}\right)$, $\left(\mathrm{R}_{\mathbf{2}}\right)$ at every $c \in(a, b]$.

THEOREM 2.2. Suppose that $a C^{1} G$-function $f: M \rightarrow R$ satisfies $\mathrm{R}(a, b)$ for $-\infty<a<b \leqq \infty$, and that $f(K)$ is bounded above if $b=\infty$. Given a family $\left\{\mathscr{F}_{c} \mid c \in(a, b]\right\}$ of finite subsets $\mathscr{F}_{c}$ of $\alpha\left(K_{c}\right)$ such that $\mathscr{F}_{c}=\{\emptyset\}$ if $K_{c}=\emptyset$, there are a finite number of critical values $c_{1}, \cdots, c_{k} \in(a, b]$ such that

$$
\alpha\left(M_{a}\right) * \mathscr{I}_{c_{1}} * \cdots * \mathscr{I}_{c_{k}} \subseteq \alpha\left(M_{b}\right) .
$$

Proof. First assume $b<\infty$. Let $\varepsilon(a)$ be such an $\varepsilon>0$ as in $\left(\mathrm{R}_{0}\right)$ at $a$. For any $c \in(a, b]$ let $\varepsilon(c)$ be such an $\varepsilon>0$ as in Lemma 2.1, i.e.,

$$
\alpha\left(M_{c-\varepsilon(c)}\right) * \mathscr{F}_{c} \subseteq \alpha\left(M_{c+\varepsilon(c)}\right) \text {. }
$$

Let $V_{c}$ denote the open interval $(c-\varepsilon(c), c+\varepsilon(c))$ for any $c \in[a, b]$. Then $\left\{V_{c} \mid c \in[a, b]\right\}$ is an open covering of $[a, b]$. Since $[a, b]$ is compact, there 
are a finite number of $d_{1}, \cdots, d_{m} \in[a, b]$ such that

$$
[a, b] \subseteq V_{d_{1}} \cup \cdots \cup V_{d_{m}} \text {. }
$$

By the monotonicity property and the assumption for $\varepsilon(b)$ we have

$$
\alpha\left(M_{b}\right) \supseteqq \alpha\left(M_{b+\varepsilon(b)}\right) \supseteqq \mathscr{I}_{b} * \alpha\left(M_{b-\varepsilon(b)}\right) .
$$

$b-\varepsilon(b)$ is contained in $V_{d}$ for some $d \in\left\{d_{1}, \cdots, d_{m}\right\}$. Since $b-\varepsilon(b)<d+\varepsilon(d)$ we have

$$
\alpha\left(M_{b-\varepsilon(b)}\right) \supseteqq \alpha\left(M_{d+\varepsilon(d)}\right) \supseteqq \mathscr{F}_{d} * \alpha\left(M_{d-\varepsilon(d)}\right) .
$$

Thus we have

$$
\alpha\left(M_{b}\right) \supseteq \mathscr{F}_{b} * \mathscr{F}_{d} * \alpha\left(M_{d-\varepsilon(d)}\right) .
$$

Repeating this argument, we have

$$
\alpha\left(M_{b}\right) \supseteqq \mathscr{F}_{c_{1}} * \cdots * \mathscr{I}_{c_{k}} * \alpha\left(M_{a}\right)
$$

for some $c_{1}, \cdots, c_{k} \in(a, b]$. If $c$ is not a critical value then $\mathscr{F}_{c}=\{\emptyset\}$ and so we may assume that $c_{1}, \cdots, c_{k}$ in (2.3) are all critical values. Thus the theorem is proved for the case $b<\infty$.

Now assume $b=\infty$. Take an $r>0$ such that $\sup f(K)<r<\infty$. By the above argument we see that there are a finite number of critical values $c_{1}, \cdots, c_{k} \in$ $(a, r]$ such that

$$
\alpha\left(M_{a}\right) * \mathscr{F}_{c_{1}} * \cdots * \mathscr{F}_{c_{k}} \subseteq \alpha\left(M_{r}\right) .
$$

Since there are no critical values in $[r, \infty)$ we can see by $\left(\mathrm{R}_{2}\right)$ that there is a $G$-map $M_{b}=M \rightarrow M_{r}$. Thus $\alpha\left(M_{r}\right)=\alpha\left(M_{b}\right)=\alpha(M)$. Thus the theorem is also proved for the case $b=\infty$.

COROLLARY 2.4. If, in Theorem 2.2, the critical values of $f$ are $c_{1}, \cdots, c_{m}$ in $(a, b]$, then

$$
\alpha\left(M_{a}\right) * \alpha\left(K_{c_{1}}\right) * \cdots * \alpha\left(K_{c_{m}}\right) \subseteq \alpha\left(M_{b}\right) .
$$

Proof. Let $\mathscr{I}_{\imath}$ be an arbitrary finite subset of $\alpha\left(K_{c_{i}}\right)$ for $1 \leqq i \leqq m$. By Theorem 2.2 we have

$$
\alpha\left(M_{a}\right) * \mathscr{I}_{\imath_{1}} * \cdots * \mathscr{F}_{\imath_{k}} \leqq \alpha\left(M_{b}\right)
$$

for some $1 \leqq i_{1}, \cdots, i_{k} \leqq m$. This implies

$$
\alpha\left(M_{a}\right) * \mathscr{F}_{1} * \cdots * \mathscr{I}_{m} \subseteq \alpha\left(M_{b}\right) .
$$

Since $\mathscr{F}_{\imath}$ is an arbitrary finite subset of $\alpha\left(K_{c_{i}}\right)$, we have

$$
\alpha\left(M_{a}\right) * \alpha\left(K_{c_{1}}\right) * \cdots * \alpha\left(K_{c_{m}}\right) \subseteq \alpha\left(M_{b}\right) .
$$


If, in Corollary 2.4, we take $b=\infty$ and if $f: M \rightarrow \boldsymbol{R}$ is bounded below, then we have

$$
\alpha\left(K_{c_{1}}\right) * \cdots * \alpha\left(K_{c_{m}}\right) \cong \alpha(M) .
$$

This means that if there are $G$-maps $K_{c_{i}} \rightarrow S_{i}\left(S_{i} \in \mathcal{A}, 1 \leqq i \leqq m\right)$, then there is a $G$-map $M \rightarrow S_{1} * \cdots * S_{m}$. The special case in which $G$ is finite and its action on $M$ is free is discussed in R.-Nowakowska [6].

\section{Numerical genera}

Let $\mathcal{S}$ be a subset of $\mathcal{A}$. As in Introduction, the numerical $\mathcal{S}$-genus $\beta_{S}(X)$ of a $G$-space $X$ is defined to be the smallest number $n$ such that there exists a $G$-map $X \rightarrow A_{1} * \cdots * A_{n}$ for $A_{i} \in \mathcal{S}(1 \leqq i \leqq n)$. If there does not exist such a $G$ map we define $\beta_{S}(X)=\infty$, and if $X=\emptyset$ then define $\beta_{\mathcal{S}}(X)=0$.

Clapp-Puppe $[2 ; \S 2]$ defined the $\mathcal{S}$-category denoted $\mathcal{S}$-cat $(f)$ for a $G$-map $f:\left(X, X^{\prime}\right) \rightarrow\left(Y, Y^{\prime}\right)$. If $c_{X}: X \rightarrow$ pt is the constant map, then $\mathcal{S}$-cat $\left(c_{X}\right)$ coincides with our $\beta_{S}(X)$ (see [2; Proposition 2.4]).

If we take as $\mathcal{S}$ the set $\mathscr{H}$ or $\mathcal{O}(X)$ given in Introduction, we have two genera $\beta_{\mathscr{H}}(X)$ and $\beta_{\mathcal{O}(X)}(X)$. Let $\gamma(X)$ denote $\beta_{\mathcal{O}(X)}(X)$. We easily see that if $X^{G}=\emptyset$ then $\beta_{\mathscr{H}}(X) \leqq \gamma(X)$, and if $X^{G} \neq \emptyset$ then $\beta_{\mathscr{H}}(X)=\infty \quad \gamma(X)=1$.

Theorem 3.1. Suppose that a $C^{1} G$-function $f: M \rightarrow \boldsymbol{R}$ satisfies $\mathrm{R}(a, b)$ for $-\infty<a<b \leqq \infty$. If $K_{(a, b]}=K \cap f^{-1}(a, b]$ consists of a finite number of orbits, then

$$
\gamma\left(M_{b}\right)-\gamma\left(M_{a}\right) \leqq \# f\left(K_{(a, b]}\right) \cdot \# \mathcal{I}\left(K_{(a, b]}\right) .
$$

If, moreover, $K_{(a, b]}^{G}=\emptyset$, then

$$
\beta_{\mathscr{H}}\left(M_{b}\right)-\beta_{\mathscr{H}}\left(M_{a}\right) \leqq \# f\left(K_{(a, b]}\right) \cdot \# \mathcal{G}\left(K_{(a, b]}\right) .
$$

Here $K_{(a, b]}^{G}$ denotes the $G$-fixed point set of $K_{(a, b]}$.

Proof. By the assumption, $m=\# f\left(K_{(a, b]}\right)<\infty$ and $n=\# g\left(K_{(a, b]}\right)<\infty$. Let $c_{1}, \cdots, c_{m}$ be the critical values in $(a, b]$. We obtain from Corollary 2.4,

and

$$
\gamma\left(M_{b}\right)-\gamma\left(M_{a}\right) \leqq \gamma\left(K_{c_{1}}\right)+\cdots+\gamma\left(K_{c_{m}}\right),
$$

$$
\beta_{\mathscr{H}}\left(M_{b}\right)-\beta_{\mathscr{H}}\left(M_{a}\right) \leqq \beta_{\mathscr{H}}\left(K_{c_{1}}\right)+\cdots+\beta_{\mathscr{H}}\left(K_{c_{m}}\right) .
$$

We see for each $1 \leqq i \leqq m, \gamma\left(K_{c_{i}}\right) \leqq n$, and $\beta_{\mathscr{H}}\left(K_{c_{i}}\right) \leqq n$ if $K_{c_{i}}^{G}=\emptyset$, and obtain

and

$$
\gamma\left(M_{b}\right)-\gamma\left(M_{a}\right) \leqq m n,
$$

$$
\beta_{\mathscr{H}}\left(M_{b}\right)-\beta_{\mathscr{H}}\left(M_{a}\right) \leqq m n \quad \text { if } \quad K_{(a, b]}^{G}=\emptyset .
$$

We conclude with some remarks. Our genera $\beta_{\mathfrak{H}}(X)$ and $\gamma(X)$ are respecti- 
vely the same as $\tilde{\gamma}_{G}(X)$ and $\gamma_{G}(X)$ in his notation of Bartsch [1]. There he gives lower bounds for these genera of some $G$-spaces where $G$ is a cyclic group $\boldsymbol{Z}_{n}$. Clapp-Puppe [2] also gives lower bounds for some $G$-spaces and the exact values for representation spheres of $G$ where $G$ is a $p$-torus $\boldsymbol{Z}_{p} \times \cdots$ $\times \boldsymbol{Z}_{p}$ with $p$ a prime, or a real torus $S^{1} \times \cdots \times S^{1}$.

\title{
REFERENCES
}

[1] T. BARTSCH, On the genus of representation spheres, Comment. Math. Helv., 65 (1990), 85-95.

[2] M. Clapp AND D. Puppe, Critical point theory with symmetries, J. Reine Angew. Math., 418 (1991), 1-29.

[3] W. KRAWCEWICZ AND W. MarzanTOWICZ, Lusternik-Schnirelman method for functionals invariant with respect to a finite group action, J. Differential Equations, 85 (1990), 105-124.

[4] M. Murayama, On $G$-ANR's and their $G$-homotopy types, Osaka J. Math., 20 (1983), 479-512.

[5] R.S. PALAIs, Lusternik-Schnirelman category on Banach manifolds, Topology, 5 (1966), 115-132.

[6] D. R.-Nowakowska, Equivariant maps of joins of finite G-sets and an applications to critical point theory, Ann. Polon. Math., 56 (1992), 195-211.

\author{
Department of Mathematics \\ YAMAGUCHI UNIVERSITY \\ YAMAGUCHI 753 \\ JAPAN \\ e-mail : komiya@ccy.yamaguchi-u.ac.jp
}

